\title{
A PRODUCT EXPANSION IN $p$-ADIC AND OTHER NON-ARCHIMEDEAN FIELDS
}

\author{
ARNOLD KNOPFMACHER AND JOHN KNOPFMACHER
}

(Communicated by Larry J. Goldstein)

ABSTRACT. An algorithm is introduced and shown to lead to a unique infinite product representation for a given $p$-adic integer $A$ with leading coefficient 1 as a product

$$
A=\prod_{n=1}^{\infty}\left(1+b_{n} p^{r_{n}}\right)
$$

where $1 \leq b_{n} \leq p-1, r_{n} \in \mathbf{N}$ and $r_{n+1}>r_{n}$. The degree of approximation by the natural number $\left(1+b_{1} p^{r_{1}}\right) \cdots\left(1+b_{n} p^{r_{n}}\right)$ is also considered. In addition we derive similar representations for elements of arbitrary complete non-Archimedean fields with discrete valuations.

1. Introduction. Let $\mathbf{Q}$ be the field of rational numbers, $p$ a prime number, and $\mathbf{Q}_{p}$ the completion of $\mathbf{Q}$ with respect to the $p$-adic valuation ||$_{p}$ defined on $\mathbf{Q}$ by

$$
|A|_{p}= \begin{cases}p^{-\alpha} & \text { if } A=p^{\alpha}(r / s) \text { where } p \nmid r, p \nmid s \\ 0 & \text { if } A=0 .\end{cases}
$$

$\mathbf{Q}_{p}$ is the field of $p$-adic numbers with $p$-adic valuation ||$_{p}$, the extension of the original valuation on $\mathbf{Q}$, which has the properties

$$
\begin{gathered}
|A|_{p} \geq 0, \quad|A|_{p}=0 \quad \text { iff } A=0 \\
|A B|_{p}=|A|_{p} \cdot|B|_{p} \\
|A+B|_{p} \leq \max \left(|A|_{p},|B|_{p}\right)
\end{gathered}
$$

with equality when $|A|_{p} \neq|B|_{p}$.

It is familiar that the above non-Archimedean valuation leads to an ultrametric distance function $\rho$ on $\mathbf{Q}_{p}$, with $\rho(A, B)=|A-B|_{p}$, making $\mathbf{Q}_{p}$ into a complete metric space with respect to $\rho$. As is common, we define the order $\nu(A)$ of $A$ by $|A|_{p}=p^{-\nu(A)}$, and set $\nu(0)=+\infty$. Then

$$
\begin{gathered}
\nu(A B)=\nu(A)+\nu(B), \quad \nu(A / B)=\nu(A)-\nu(B) \quad \text { if } B \neq 0, \\
\nu(A+B) \geq \min (\nu(A), \nu(B))
\end{gathered}
$$

with equality when $\nu(A) \neq \nu(B)$. Our main attention below will be confined to those $p$-adic numbers lying in the open unit ball $\mathscr{P}_{1}=1+p \mathbf{Z}_{p}$ with centre 1 , i.e. the $p$-adic integers which have $p$-adic expansions of type $1+\sum_{n=1}^{\infty} c_{n} p^{n}, 0 \leq c_{n} \leq p-1$.

Our main result is:

Received by the editors May 15, 1987 and, in revised form, January 4, 1988.

1980 Mathematics Subject Classification (1985 Revision). Primary 10F45, 12B40. 
(1.4) THEOREM. Every $A \in \mathscr{P}_{1}$ with $A \neq 1$ has a unique convergent product representation (relative to $\rho$ ) of the form

$$
A=\prod_{n=1}^{\infty}\left(1+b_{n} p^{r_{n}}\right)
$$

where $1 \leq b_{n} \leq p-1, r_{n} \in \mathbf{N}$ and $r_{n+1}>r_{n}$. Further $\nu\left(A-P_{n}\right) \geq r_{n+1}>n$ where $P_{n}=\left(1+b_{1} p^{r_{1}}\right) \cdots\left(1+b_{n} p^{r_{n}}\right) \in \mathbf{N}$.

This theorem is quite analogous to one on $p$-adic continued fractions of the form.

$$
A=\frac{p^{r_{1}}}{b_{1}}+\frac{p^{r_{2}}}{b_{3}}+\frac{p^{r_{3}}}{b_{2}}+\cdots,
$$

which were originally proposed by Mahler [3], and studied further by Schneider [5] and Bundschuh $[\mathbf{1}]$.

In the final section, a few remarks are made about extensions to a general complete non-Archimedean field with a discrete valuation.

2. Convergence and approximation. If $1 \neq A \in \mathscr{P}_{1}$, let $\hat{A}_{1}=A$. Then we can write

$$
\hat{A}_{1}=1+b_{1} p^{r_{1}} A_{1}^{\prime},
$$

where $r_{1} \in \mathbf{N}, 1 \leq b_{1} \leq p-1$ and $A_{1}^{\prime} \in \mathscr{P}_{1}$. If $\hat{A}_{n}=1+b_{n} p^{r_{n}} A_{n}^{\prime}$ has already been defined with $1 \neq A_{n}^{\prime} \in \mathscr{P}_{1}, 1 \leq b_{n} \leq p-1$, then define

$$
\begin{aligned}
\hat{A}_{n+1} & =\left(1+b_{n} p^{r_{n}}\right)^{-1} \hat{A}_{n}=\left(1-b_{n} p^{r_{n}}+b_{n}^{2} p^{2 r_{n}}-\cdots\right)\left(1+b_{n} p^{r_{n}}+\cdots\right) \\
& =1+b_{n+1} p^{r_{n+1}} A_{n+1}^{\prime},
\end{aligned}
$$

where $A_{n+1}^{\prime} \in \mathscr{P}_{1}, r_{n+1}>r_{n}$ and $1 \leq b_{n+1} \leq p-1$.

If $A_{n}^{\prime}=1$, let $A_{n+1}^{\prime}=1$ and stop the algorithm.

Then

$$
A=\hat{A}_{1}=\left(1+b_{1} p^{r_{1}}\right) \hat{A}_{2}=\cdots=\hat{A}_{n+1} \prod_{i=1}^{n}\left(1+b_{i} p^{r_{i}}\right) .
$$

If the procedure does not terminate with some $\hat{A}_{n+1}=1$, then

$$
\nu\left(\hat{A}_{n+1}-1\right)=r_{n+1} \geq 1+r_{n} \geq \cdots \geq n+1 \rightarrow \infty \text { as } n \rightarrow \infty .
$$

Thus $\lim _{n \rightarrow \infty} \hat{A}_{n+1}=1$, and then $A=\prod_{i=1}^{\infty}\left(1+b_{i} p^{r_{i}}\right)$, relative to the metric $\rho$ on $\mathbf{Q}_{p}$. If $P_{n}=\left(1+b_{1} p^{r_{1}}\right) \cdots\left(1+b_{n} p^{r_{n}}\right)$, we also have

$$
A=\left(1+b_{n+1} p^{r_{n+1}} A_{n+1}^{\prime}\right) P_{n}
$$

and so $\nu\left(A-P_{n}\right)=\nu\left(b_{n+1} p^{r_{n+1}} A_{n+1}^{\prime} P_{n}\right)=r_{n+1}>n$.

\section{Uniqueness of representation.}

(3.1) LEMMA. Any product $\prod_{n \geq 1}\left(1+d_{n} p^{s_{n}}\right)$ with $1 \leq d_{n} \leq p-1, s_{n} \in \mathbf{N}$ and $s_{n+1}>s_{n}$, converges relative to $\rho$ to an element $B \neq 1$ in $\mathscr{P}_{1}$ with $B=1+d_{1} p^{s_{1}} B^{\prime}$, $B^{\prime} \in \mathscr{P}_{1}$.

PROOF. If $Q_{N}=\prod_{n=1}^{N}\left(1+d_{n} p^{s_{n}}\right)$, then

$$
\begin{gathered}
Q_{N+K}-Q_{N}=Q_{N} R_{N, K}, \text { where } \\
R_{N, K}=\prod_{n=N+1}^{N+K}\left(1+d_{n} p^{s_{n}}\right)-1=d_{N+1} p^{s_{N+1}} R_{N, K}^{\prime}
\end{gathered}
$$


for $R_{N, K}^{\prime} \in \mathscr{P}_{1}$, since $\left(s_{N}\right)$ is a strictly increasing sequence. Thus

$$
\nu\left(Q_{N+K}-Q_{N}\right)=\nu\left(Q_{N}\right)+\nu\left(R_{N, K}\right)=s_{N+1}>N \rightarrow \infty
$$

as $N \rightarrow \infty$, independently of $K \geq 1$. Therefore, if the product is infinite, then $\left(Q_{N}\right)$ forms a Cauchy sequence and converges relative to $\rho$ to an element $B$ of the complete metric space $\mathbf{Q}_{p}$. Since the convergence is relative to $\rho, \nu\left(B-Q_{N}\right)>s_{1}$ for $N$ sufficiently large, and so $B=1+d_{1} p^{s_{1}} B^{\prime}$ for $B^{\prime} \in \mathscr{P}_{1}$, because $Q_{N}=1+R_{0, N}=$ $1+d_{1} p^{s_{1}} R_{0, N}^{\prime}$. Now suppose that $A=\prod_{n \geq 1}\left(1+b_{n} p^{r_{n}}\right)=\prod_{n \geq 1}\left(1+d_{n} p^{s_{n}}\right)$, where $1 \leq b_{n} \leq p-1,1 \leq d_{n} \leq p-1, r_{n}, s_{n} \in \mathrm{N}$ and $r_{n+1}>r_{n}, s_{n+1}>s_{n}$. Then Lemma 3.1 implies that

$$
A=1+b_{1} p^{r_{1}} A^{\prime}=1+d_{1} p^{s_{1}} B^{\prime} \quad \text { for } A^{\prime}, B^{\prime} \in \mathscr{P}_{1} .
$$

Hence $b_{1}=d_{1}, r_{1}=s_{1}$, and we obtain $\prod_{n \geq 2}\left(1+b_{n} p^{r_{n}}\right)=\prod_{n \geq 2}\left(1+d_{n} p^{s_{n}}\right)$. In the same way, $b_{2}=d_{2}$ and $r_{2}=s_{2}$, and thus we successively obtain $b_{n}=d_{n}, r_{n}=s_{n}$ for all $n$.

REMARKS. It is interesting to note from the general form of the product for $A$, and from simple examples within $\mathbf{Q}_{p}$, such as

$$
1+3 p+2 p^{2}=(1+3 p)\left(1+2 p^{2}\right)\left(1+4 p^{3}\right) \cdots, \quad \text { if } p=5 \text { say }
$$

that "most" natural numbers have infinite representations as in Theorem 1.4, the exceptions being those of the general type $P_{n}$ of Theorem 1.4. For natural numbers of type $P_{n}$, the above algorithm then provides an actual method for factorizing $P_{n}$ in the indicated special way.

A study of the relationship between properties of the $p$-adic product coefficients and properties of $A$ or of its $p$-adic series coefficients might perhaps prove interesting. As two little comments in this direction, we note:

(i) If $A=1+b_{1} p^{r_{1}}+b_{2} p^{r_{2}}+\cdots$, where $r_{1}<r_{2}, 1 \leq b_{i} \leq p-1$, then $A=$ $\left(1+b_{1} p^{r_{1}}\right)\left(1+b_{2} p^{r_{2}}\right) \cdots$, where however the subsequent factors of the product need not necessarily conform to this initial pattern.

(ii) For a simple rational number $\left(1-p^{r}\right)^{-1}$, with $r \in \mathbf{N}$, the coefficients in the product are given explicitly by the Euler-type identity which occurs in the proof of Proposition 4.1 below.

4. Product expansions for rational numbers. We note that there remains the possibly interesting problem of characterizing the types of products in Theorem 1.4 above which correspond to rational numbers $A \in \mathbf{Q}$. In the analogous case of the $p$-adic continued fractions studied by Schneider [5] and Bundschuh [1], rational numbers are characterized by finite or certain periodic continued fractions. However, since finite expansions as in Theorem 1.4 yield natural numbers, a characterization of nonintegral rationals as well as most natural numbers would therefore have to involve infinite products, satisfying possibly special conditions on the exponents $r_{n}$. As a partial result in this direction we note:

(4.1) Proposition. Let $A \in \mathscr{P}_{1}, A \neq 1$, with unique product representation

$$
A=\prod_{n=1}^{\infty}\left(1+b_{n} p^{r_{n}}\right)
$$


If $b_{n}=1$ and $r_{n+1}=2 r_{n}$, for all $n$ sufficiently large, then $A \in \mathbf{Q}$.

PROOF. This follows easily from the Euler-type identity

$$
\prod_{n=1}^{\infty}\left(1+y^{2^{n-1}}\right)=\frac{1}{1-y} \text { where } y=p^{r}, r \in \mathbf{N} .
$$

To establish this identity, note that the equation $1+y=\left(1-y^{2}\right) /(1-y)$ leads to

$$
\prod_{n=1}^{N}\left(1+y^{2^{n-1}}\right)=\frac{1-y^{2}}{1-y} \cdots \frac{1-y^{2^{N}}}{1-y^{2^{N-1}}}=\frac{1-y^{2^{N}}}{1-y}
$$

The identity then follows, since $\nu\left(y^{k}\right)=k r \rightarrow \infty$ as $k \rightarrow \infty$, i.e. $y^{k} \rightarrow 0$ relative to $\rho$.

5. Products in arbitrary discrete-valued non-Archimedean fields. Let $(K,||)$ represent a complete non-Archimedean valued field. Thus the norm || of $K$ satisfies properties equivalent to those of equation (1.2) for ||$_{p}$. We define the function $\nu$, the valuation of $K$ by

$$
\nu(A)= \begin{cases}-\log |A| & \text { if } A \in K, A \neq 0, \\ +\infty & \text { if } A=0 .\end{cases}
$$

Then $\nu$ has the same properties as those in equation (1.3) for the $p$-adic valuation. As previously, we can define an ultrametric distance function $\rho$ on $K$, with $\rho(A, B)=|A-B|$. Let $K^{\times}$denote the set $K^{\times}=\{A \in K, A \neq 0\}$ and for a subset $X \subset K$ let $|X|=\{|A|: A \in X\}$. The value group of $K$ is the subgroup $\left|K^{\times}\right|$of the multiplicative group of the positive real numbers. Suppose now $K$ has a discrete valuation. Then (see e.g. Schikhof [4]) there is an element $\pi \in K$ such that

$$
|\pi|=\max \left|K^{\times}\right| \cap(0,1)
$$

and

$$
\left|K^{\times}\right|=\left\{|\pi|^{n}: n \in \mathbf{Z}\right\} \text {. }
$$

The element $\pi$ of $K$ plays a role similar to that of $p$ in $\mathbf{Q}_{p}$. Next we define the residue class field of $K$ to be the field

$$
k=B_{0}(1) / B_{0}\left(1^{-}\right)=\{A \in K:|A| \leq 1\} /\{A \in K:|A|<1\}
$$

where $B_{0}(1)$ and $B_{0}\left(1^{-}\right)$denote the closed and open unit discs with centre 0 , respectively, and let $R$ denote a full set of representatives for $k$ in $K$. Thus $R$ is a subset of the closed unit disc $B_{0}(1)$ of $K$ which satisfies

(i) if $r_{1}, r_{2} \in R, r_{1} \neq r_{2}$ then $\left|r_{1}-r_{2}\right|=1$;

(ii) for every $A \in B_{0}(1)$ there is an $r \in R$ such that $|A-r|<1$. In the case $K=\mathbf{Q}_{p}$ the set $R=\{0,1, \ldots, p-1\}$ satisfies (i) and (ii) above. In general $R$ may be an infinite set.

With the above notation and assuming for simplicity that $0 \in R$, it can be shown (Theorem 12.1, Schikhof [4]) that every $A \in K$ has a unique series representation

$$
A=\sum_{j=-n}^{\infty} a_{j} \pi^{j}, \quad \text { some } n \in \mathbf{N}, a_{j} \in R .
$$


Consider now the subset $\mathscr{P}_{1}$ of $K$ consisting of all elements in the 'open' unit ball $B_{1}\left(1^{-}\right)$, that is those elements of $K$ having series expansion of the form

$$
A=1+\sum_{j=1}^{\infty} a_{j} \pi^{j}, a_{j} \in R .
$$

In an entirely analogous way to the case $K=\mathbf{Q}_{p}$ we can now prove:

(5.1) THEOREM. Every $A \in \mathscr{P}_{1}$, with $A \neq 1$, has a unique convergent product representation (relative to $\rho$ ) of the form

$$
A=\prod_{n=1}^{\infty}\left(1+b_{n} \pi^{r_{n}}\right),
$$

where $0 \neq b_{n} \in R, r_{n} \in \mathbf{N}$ and $r_{n+1}>r_{n}$. Further, $\nu\left(A-P_{n}\right) \geq r_{n-1}>n$, where $P_{n}=\left(1+b_{1} \pi^{r_{1}}\right) \cdots\left(1+b_{n} \pi^{r_{n}}\right)$.

Other earlier results, such as the Euler-type identity in the proof of Proposition 4.1 for example, also extend to the present setting. One case of special relevance to complex analysis is treated by us more fully elsewhere [2].

\section{REFERENCES}

1. P. Bundschuh, $p$-adische Kettenbrüche und Irrationalität p-adischer Zahlen, Elem. Math. 32 (1977), 36-40.

2. A. Knopfmacher and J. Knopfmacher, Infinite products for power series, J. Approx. Theory (to appear).

3. K. Mahler, Zur Approximation p-adischer Irrationalzahlen, Nieuw Arch. Wisk. 18 (1934), 2234.

4. W. H. Schikhof, Ultrametric calculus, Cambridge Univ. Press, 1984.

5. Th. Schneider, Über p-adische Kettenbrüche, Symposia Mat. IV (1970), 181-189.

Department of Applied Mathematics, University of THE WitwatersRand, JOHANNESBURG, SOUTH AFriCA, Wits 2050

DEPARTMENT OF MATHEMATICS, UNIVERSITY OF THE WITWATERSRAND, JOHANNESBURG, SOUTH AFrICA, Wits 2050 